\title{
Application of spherical harmonics analysis on LBS particles and LBS frag- ments
}

\author{
Budi Zhao ${ }^{1, \star}$, Deheng $\mathrm{Wei}^{1}$, and Jianfeng Wang ${ }^{1}$ \\ ${ }^{1}$ Department of Architecture and Civil Engineering, City University of Hong Kong, Hong Kong
}

\begin{abstract}
This paper applies surface parameterization and spherical harmonics analysis to the characterization of particle shapes of Leighton Buzzard sand (LBS) particles and LBS fragments obtained from X-ray microtomography $(\mu \mathrm{CT})$. The rotation, transition and scale independent spherical coefficients were obtained. The relationship between spherical coefficients and shape parameters of form, roundness and compactness was investigated. The coefficients of degree one determine the principal dimensions of an ellipsoid, which has a similar aspect ratio with the original surface. The coefficients of higher degree characterise more details by increasing the percentage of higher and lower mean curvature on the reconstructed surface. As the spherical degree increases, the reconstructed surface tend to have lower particle roundness, sphericity and convexity, and higher aspect ratio.
\end{abstract}

\section{Introduction}

The studies on particle shape characterization and reconstruction mainly have two objectives. The first one is to classify and quantify particle shapes. It could be used to relate shape characteristics with performance properties (e.g. mechanical behaviour of granular materials) [1] and understand the broad class of phenomena that involve shape evolution (e.g. weathering and fragmentation) [24]. The simplest way to obtain shape parameters (e.g. aspect ratio, roundness and compactness) is to form dimensionless indices with principal dimensions, surface area, volume or local curvature. The second objective is to randomly generate shapes for numerical simulation models. Fourier transformation and spherical harmonics method could mathematically reconstruct and randomly generate particle shapes in two-dimension and three-dimension, respectively $[5,6]$.

This study investigates three-dimensional (3D) particle shape characterization with spherical harmonics analysis. Leighton Buzzard sand (LBS) particles and LBS fragments were analyzed. The transition, rotation and size independent coefficients were obtained. The influence of spherical coefficients on particle form, roundness and compactness was investigated.

\section{Shape description using spherical harmonics}

Spherical harmonics are a complete set of orthogonal functions defined on the surface of a sphere. It has been widely used to mathematically represent $3 \mathrm{D}$ particle shapes. It

\footnotetext{
^e-mail: bdzhao2-c@my.cityu.edu.hk
}

originally used radial representation $(r(\theta, \phi))$ which is restricted to star-shaped surfaces [7]. This limitation was overcome by a surface parameterization, which defines a continuous, one-to-one mapping from the surface of the original object to a unit sphere [8].

\subsection{Spherical surface parameterization}

Parameterization could be considered as a constrained optimization problem that embeds the object surface onto the surface of a unit sphere while minimizing the distortion of the surface net. Earlier parameterization approaches used voxel assembly of objects [8]. It has been recently extended to triangular meshes [9]. In this study, we follows the Control of Area and Length Distortions (CALD) framework proposed in [9].

In the CALD algorithm, a bijective mapping was obtained between each vertex on a surface $\mathbf{v}=(x, y, z)$ and a pair of coordinates $\theta$ and $\phi . \theta \in[0, \pi]$ and $\phi \in[0,2 \pi)$ are the latitudinal and longitudinal coordinate, respectively. The $\theta$ and $\phi$ values of each vertice were determined mainly through local smoothing method and global smoothing method to reduce the area and length distortions. It is performed on each component of $\mathbf{v}$ independently, which resulted in: $\mathbf{v}(\theta, \phi)=(x(\theta, \phi), y(\theta, \phi), z(\theta, \phi))^{T}$. An illustration of the surface parameterization is shown in figure 1(b).

\subsection{Spherical harmonics transformation}

Spherical harmonics analysis is generally seen as the extension of Fourier analysis on the unit sphere. Any spherical scaler function $(f(\theta, \phi))$ could be decomposed as the 


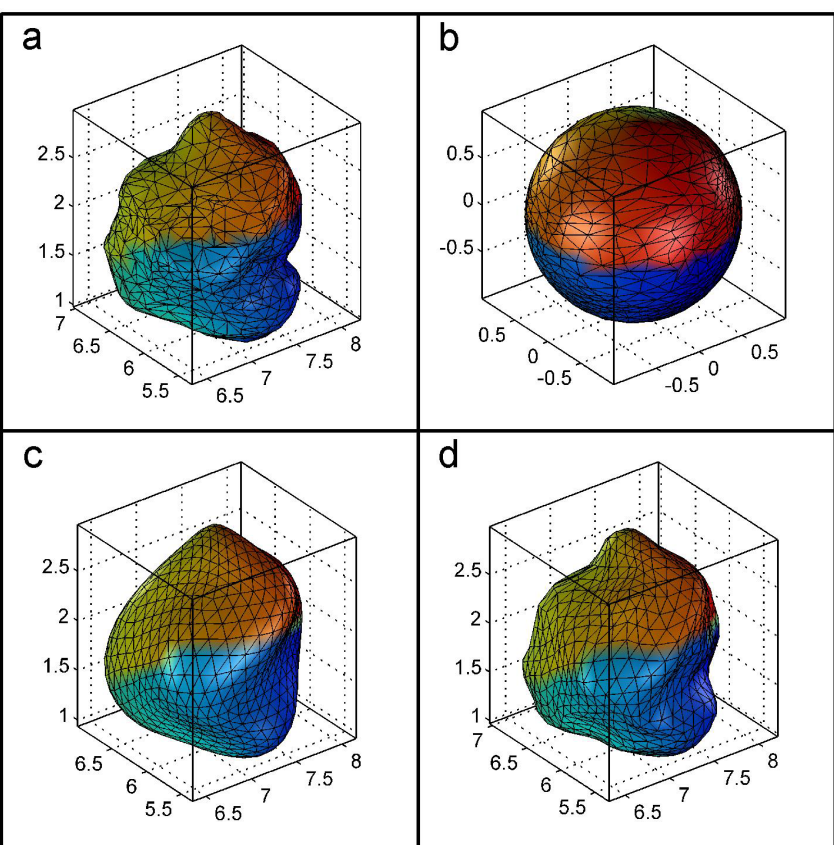

Figure 1. Surface parameterization and reconstruction using SPHARM-MAT toolbox: (a) original surface; (b) mapping on the unit-sphere; (c) reconstruction using $l=5$; (d) reconstruction using $l=10$

sum of spherical harmonics:

$$
f(\theta, \phi)=\sum_{l=0}^{\infty} \sum_{m=-l}^{m=l} c_{l}^{m} Y_{l}^{m}(\theta, \phi)
$$

where $Y_{l}^{m}$ and $c_{l}^{m}$ are called the spherical harmonic function and coefficient of degree $l$ and order $m$. The set of spherical harmonics $Y_{l}^{m}$ are called Laplace's spherical harmonics, which form an orthonormal basis of the Hilbert space of square-integrable functions:

$$
Y_{l}^{m}(\theta, \phi)=\frac{(2 l+1)}{4 \pi} \frac{(l-m) !}{(l+m) !} P_{l}^{m}\left(\cos \theta e^{i m \phi}\right)
$$

where $P_{l}^{m}$ is the associated Legendre polynomial.

The spherical harmonics analysis is performed on each component of $\mathbf{v}(\theta, \phi)$ independently, which resulted in harmonic coefficients with three components, $\mathbf{c}_{1}^{m}=$ $\left(c_{x l}^{m}, c_{y l}^{m}, c_{z l}^{m}\right)^{T}$. The coefficients $\mathbf{c}_{l}^{m}$ up to a user-defined degree can be estimated by solving a set of linear equations in a least square fashion. Figure 1(c,d) shows the reconstruction with different spherical degrees.

\subsection{Rotation, transition and size-independent coefficients}

The coefficients $\left(\mathbf{c}_{l}^{m}\right)$ obtained so far depend not only on particle shape, but also on the relative position, orientation and size of a particle. The rotational invariance was obtained by considering the contribution of the spherical harmonics of degree $l=1$, an ellipsoid. It involves rotating the object in both parameter space and object space. The parameter space is rotated so that the north pole $(\theta=0)$

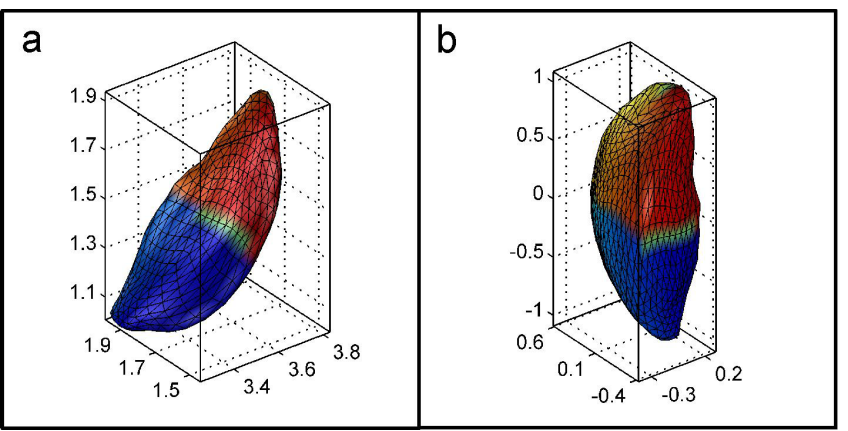

Figure 2. Surface alignment using SPHARM-MAT toolbox: (a) original surface; (b) surface after translation, rotation and scaling

will be at one end of the shortest main axis of this firstorder ellipsoid and the point where the Greenwich meridian $(\phi=0)$ crosses the equator $(\theta=\pi / 2)$ is at one end of the longest main axis. Then, the ellipsoid is rotated in the object space to make its main axes coincide with the coordinate axes. The resulted coefficients are invariant under rotation of the object, except mirroring.

The transitional invariance could be achieved by ignoring $\mathbf{c}_{0}^{0}$. Scaling invariance can be achieved by dividing all coefficients by $\sqrt[3]{V}$, where $V$ is the volume of the particle. The scaled particles have a unit volume of $1 \mathrm{~mm}^{3}$. The resulted coefficients $\left(\mathbf{c}_{l}^{\prime \prime m}\right)$ are rotation, transition and scaleindependent. Figure 2 shows the original particle surface and the surface reconstructed from $\mathbf{c}^{\prime \prime m}{ }_{l}$.

\section{Application to LBS particles and LBS fragments}

The shapes of Leighton Buzzard sand (LBS) particles and LBS fragments generated from single particle crushing tests were analyzed. LBS particles and LBS fragments were scanned with X-ray micro-tomography $(\mu \mathrm{CT})$ systems with a resolution of $15 \mu \mathrm{m}$ and $3 \mu \mathrm{m}$, respectively. The LBS particles have particle size between 1 and $2 \mathrm{~mm}$, while the LBS fragments are larger than $0.001 \mathrm{~mm}^{3}$. The raw CT images were put through a series of image processing methods to obtain the smooth triangular surface mesh [3], based on which we performed the spherical harmonics analysis and shape quantification at form, roundness and compactness.

\subsection{Extraction of the spherical harmonics coefficients}

We used the SPHARM-MAT Toolbox (available at http://www.iu.edu/ shenlab/software.html) to perform the spherical parameterization and compute the spherical harmonic coefficients independent from transition, rotation and scaling.

\subsection{Shape quantification on form, roundness and compactness}

We followed the framework proposed in [3] to quantify the surface reconstructed with spherical harmonics at form, 


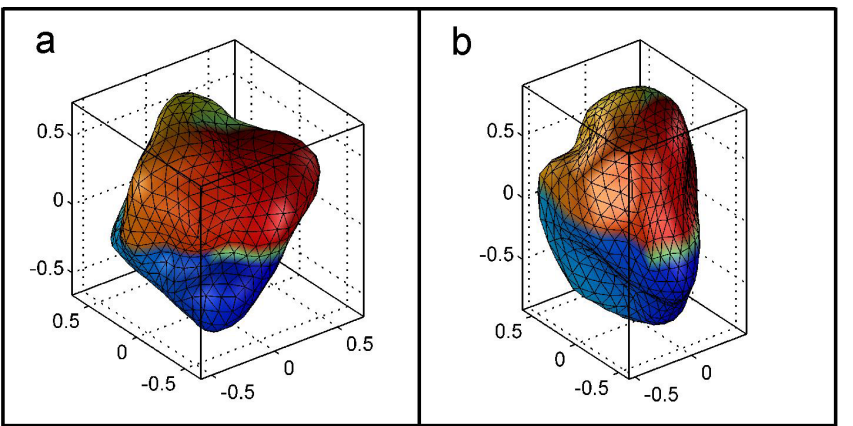

AR: $0.96 ; R_{M}: 0.65$ S: $0.88 ; C_{X}: 0.92$

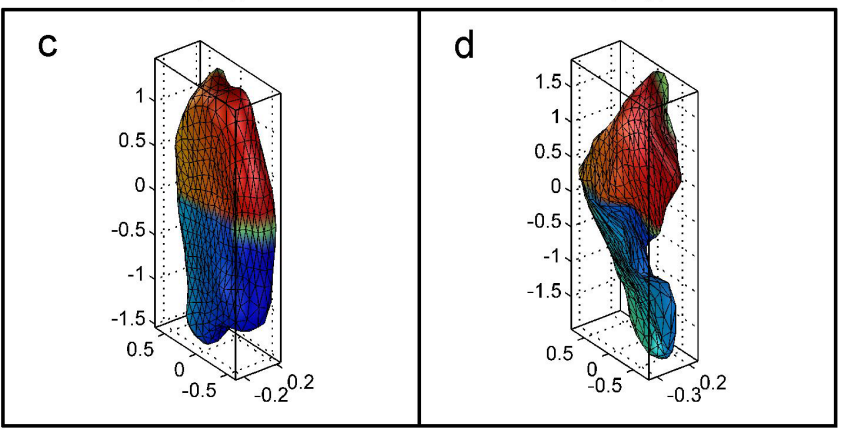

AR: $0.42 ; R_{M}: 0.80$

S: $0.65 ; C_{X}: 0.84$

AR: $0.42 ; R_{M}: 0.70$

S: $0.52 ; C_{X}: 0.59$

Figure 3. Reconstruction of LBS particles ( $a$ and $b$ ) and LBS fragments (c and d) from $\mathbf{c}^{\prime \prime m}{ }_{l}^{m}(l \leq 15)$ along with shape parameters

roundness and compactness. A 3D particle has three principal dimensions $(a \geq b \geq c)$. Two aspect ratios were defined as elongation index $(E I=b / a)$ and flatness index $(F I=c / b)$. Then, the representative aspect ratio (AR) was defined as the average value of $E I$ and $F I$. Sphericity $(S)$ was defined as the ratio between the surface area of a particle's volume-equivalent sphere and the particle. Convexity $\left(C_{X}\right)$ was defined as the ratio between the volume of the particle and the volume of its convex hull. To evaluate particle roundness, the surface was simplified with 1280 triangular meshes to represent the cut-off between roundness and roughness. Then, particle roundness index $\left(R_{M}\right)$ was defined from local mean curvature values around the corners, which were defined as the part of surface that has larger local mean curvature than its area-equivalent sphere. Area-equivalent sphere instead of maximum inscribed sphere was used to define corners due to the difficulty in obtaining the maximum inscribed sphere from triangular surface mesh. The area-equivalent sphere is always larger than the maximum inscribed sphere, which leads to larger area of identified corners.

\section{Results}

Totally 80 LBS particles and 46 LBS fragments were analyzed in this study. Figure 3 shows the reconstruction of two LBS particles and two LBS fragments along with their shape parameters. The influence of spherical coefficients
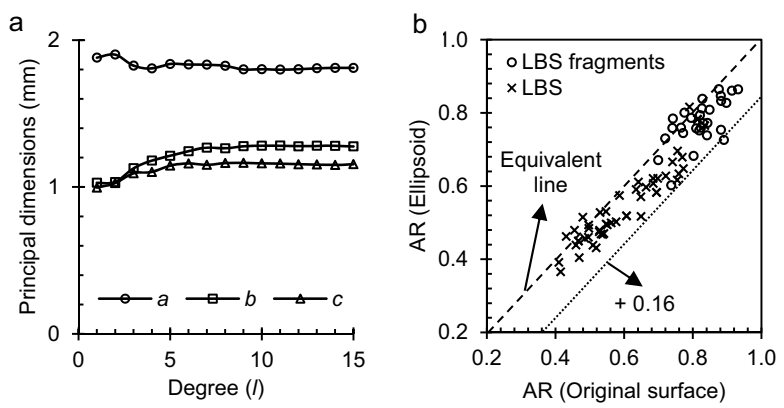

Figure 4. (a) Principal dimensions of a reconstructed LBS particle as spherical degree increases; (b) relationship between AR values of original surfaces and AR values of ellipsoids reconstructed with $\mathbf{c}_{1}^{\prime \prime m}$

on the form, roundness and compactness of reconstructed surface is described as follows.

- Particle form. As shown in figure 4(a), the maximum principal dimension $(a)$ does not change much as the degree increases, while the medium and minimum dimensions ( $b$ and $c$ ) slightly increase before degree 7 . The coefficients of degree one determine an ellipsoid. The three principal dimensions of the ellipsoid could be calculated with $a=\left\|c_{z, 1}^{\prime \prime m}\right\|, b=\left\|c_{y, 1}^{\prime \prime m}\right\|$ and $c=\left\|c_{x, 1}^{\prime \prime m}\right\|$. Figure 4(b) shows that most particles have AR values slightly larger than the ellipsoids defined by $c^{\prime \prime m}$.

- Particle roundness. Particle roundness characterises the variances around corners. It is a surface feature between particle form and roughness. A LBS particle and a LBS fragment were reconstructed with highest spherical degree of $1,5,8$ and 15 . The reconstructed surface was all represented by 1280 triangular meshes, which serve as the cut-off between roundness and roughness. Figure 5 shows the cumulative distributions of the mean curvatures on these surfaces. It is found that as the degree increases the variance of mean curvature increases, which characterises better the finer features of particle shape. For example, the proportion of surface with higher mean curvature increases.

- Particle compactness. Particle compactness describes the relationship between surface area, volume and volume of convex hull. It is thus an overall feature which is related to particle form, roundness and roughness. As the degree increases, the finer features will be better characterised, which will result in smaller particle compactness values.

The distributions of shape parameters for LBS particles and LBS fragments were fitted with Weibull's distributions with two parameters, i.e. the characteristic value $\left(L_{0}\right)$ and Weibull modulus $(m)$. The characteristic value and Weibull modulus describe the average value and the variance of shape parameters, respectively. The evolution of $L_{0}$ and $m$ for LBS particles and LBS fragments reconstructed with different spherical degrees was shown in figure 6. It is found that as the degree increases, the characteristic value $\left(L_{0}\right)$ of AR decreases, while that of $R_{M}, \mathrm{~S}$ 

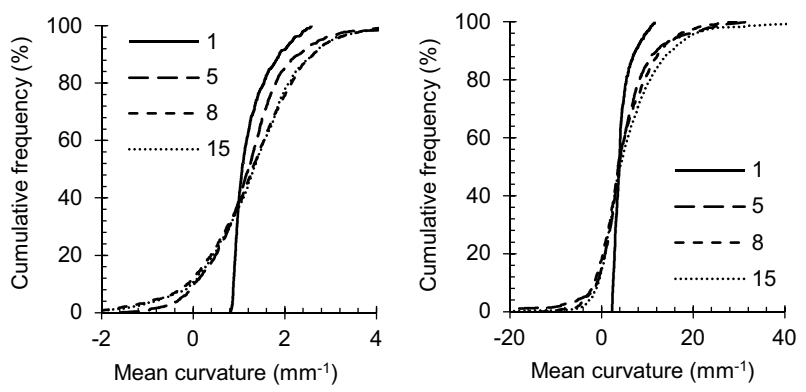

Figure 5. Distributions of mean curvature for reconstructed surface with 1,280 triangular meshes with different degrees for (a) a LBS particle and (b) a LBS fragment
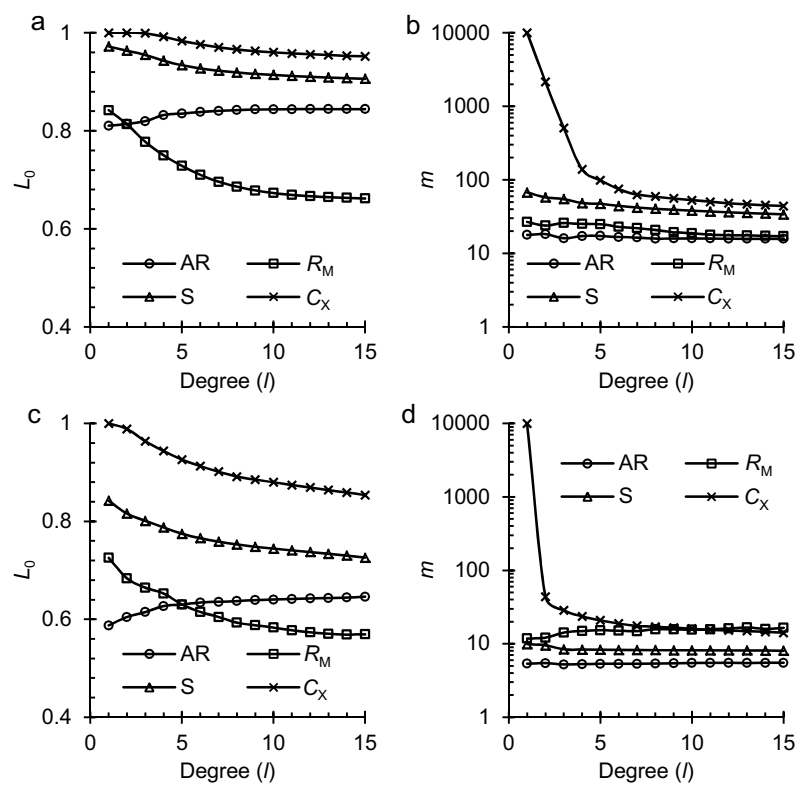

Figure 6. Variance of characteristic shape parameters $\left(L_{0}\right)$ and Weibull modulus $(m)$ for reconstructed surfaces with different spherical harmonics degree $(l)$ for (a-b) LBS particles and (c-d) LBS fragments

and $C_{X}$ decreases. It tends to converge at a higher degree. If we consider a $5 \%$ difference from the shape parameters of surface reconstructed with $\mathbf{c}^{\prime \prime \prime}{ }_{l}(l \leq 15)$ as the threshold for a good particle reconstruction, the degree needs to characterize form, roundness and compactness of both LBS particles and LBS fragments is 8 . The increase of Weibull modulus $(m)$ indicates that the variance of shape parameters increase as the degree increases, especially for convexity.

\section{Conclusions}

This study performed spherical harmonics analysis on LBS particles and LBS fragments. The reconstructed surface was quantified at form, roundness and compactness. It is found that particle form could be well described with an ellipsoid represented by coefficients of degree one. The coefficients of degrees larger than one characterise more detail surface features, which could be reflected by the variance of mean curvature distributions. In general, as the degree increases, particle form will increase; particle roundness, sphericity and convexity will decrease. The statistical analysis on the shape parameters of reconstructed surface indicates that a maximum degree of 8 is enough for characterizing the form, roundness and compactness of LBS particles and LBS fragments.

\section{Acknowledgement}

The study presented in this article was supported by General Research Fund No. CityU 120512 from the Research Grant Council of the Hong Kong SAR and Research Grant No. 51379180 from the National Science Foundation of China.

\section{References}

[1] Cho G C, Dodds J \& Santamarina J C, Journal of geotechnical and geoenvironmental engineering 132(5), 591-602 (2006)

[2] Zhao B D, Wang J F, Coop M R, Viggiani G \& Jiang M J, Géotechnique 65(8), 625-641 (2015)

[3] Zhao B D \& Wang J F, Powder technology 291, 262275 (2016)

[4] Domokos G, Kun F, Sipos A Á, \& Szabó T, Scientific reports 5, 9147 (2015)

[5] Mollon G \& Zhao J D, Granular matter 14(5), 621-638 (2012)

[6] Zhou B, Wang J F \& Zhao B D, Engineering geology 184, 126-137 (2015)

[7] Ballard D H \& Brown C M, Computer Vision (Prentice-Hall, Englewood Cliffs, NJ, 1981)

[8] Brechbühler CH, Gerig G \& Kübler, O., Computer vision and image understanding 61(2), 154-170 (1995)

[9] Shen L \& Makedon F, Image and vision computing 24(7), 743-761 (2006)

[10] Barrett P J, Sedimentology 27(3), 291-303 (1980)

[11] Bowman E T, Soga K \& Drummond W, Géotechnique, 51(6), 545-554 (2001) 